\begin{tabular}{|c|l|}
\hline Title & Liquid Droplets A ct as “ Compass Needles" for the Stresses in a Deformable Membrane \\
\hline Author(s) & Schulman, Rafael D.; Ledesma A lonso, René; Sal ez, Thomas; Raphäl, Elie; Dal noki-V eress, Kari \\
\hline Citation & $\begin{array}{l}\text { Physical Review Letters, 118, 198002-1-198002-6 } \\
\text { https://doi.org/10.1103/PhysRevLett.118.198002 }\end{array}$ \\
\hline Issue Date & 2017-05-11 \\
\hline Doc URL & http://hdl.handle.net/2115/67784 \\
\hline Rights & @2017 A merican Physical Society \\
\hline Type & article \\
\hline Additional Information & There are other files related to thisitem in HUSCAP. Check the above URL. \\
\hline Information & PhysRevLett.118.198002.pdf () \\
\hline
\end{tabular}

Instructions for use 


\title{
Liquid Droplets Act as "Compass Needles" for the Stresses in a Deformable Membrane
}

\author{
Rafael D. Schulman, ${ }^{1}$ René Ledesma-Alonso, ${ }^{2,3}$ Thomas Salez, ${ }^{3,4}$ Elie Raphaël, ${ }^{3}$ and Kari Dalnoki-Veress ${ }^{1,3, *}$ \\ ${ }^{1}$ Department of Physics and Astronomy, McMaster University, 1280 Main Street West, Hamilton, Ontario, L8S 4M1, Canada \\ ${ }^{2}$ CONACYT-Universidad de Quintana Roo, Boulevar Bahía s/n, Chetumal, 77019 Quintana Roo, México \\ ${ }^{3}$ Laboratoire de Physico-Chimie Théorique, UMR CNRS Gulliver 7083, ESPCI Paris, PSL Research University, 75005 Paris, France \\ ${ }^{4}$ Global Station for Soft Matter, Global Institution for Collaborative Research and Education, Hokkaido University, \\ Sapporo, Hokkaido 060-0808, Japan
}

(Received 1 March 2017; published 11 May 2017)

\begin{abstract}
We examine the shape of droplets atop deformable thin elastomeric films prepared with an anisotropic tension. As the droplets generate a deformation in the taut film through capillary forces, they assume a shape that is elongated along the high tension direction. By measuring the contact line profile, the tension in the membrane can be completely determined. Minimal theoretical arguments lead to predictions for the droplet shape and membrane deformation that are in excellent agreement with the data. On the whole, the results demonstrate that droplets can be used as probes to map out the stress field in a membrane.
\end{abstract}

DOI: 10.1103/PhysRevLett.118.198002

The physics of liquid droplets in contact with soft or deformable solids, elastocapillarity, is an active subject of research. Between capillary origami and wrinkling instabilities of thin films [1-9], the bending, coiling, and winding of slender structures [10-16], and elasticitymediated propulsion of droplets [17-19], there is no shortage of complexity, self-assembly, or beautiful examples of pattern formation in the field. In addition, some recent results have forced us to question familiar concepts of solid-liquid interactions. For instance, studies on the partial wetting of liquid drops on soft solids show that Young's law is applicable on length scales much larger than the bulk elastocapillary length $\gamma / E$, where $\gamma$ is the liquid-air surface tension and $E$ is the Young's modulus of the solid. However, on smaller length scales, the contact line reveals a wetting ridge set by a Neumann construction involving surface stresses [20-26].

Partial wetting on deformable substrates may also be studied by employing a highly compliant geometry, such as a droplet on a thin freestanding film [27-31]. These studies have considered clamped films which are held taut and support a uniform and isotropic tension. As shown in Fig. 1(a), the Laplace pressure of the droplet creates a bulge in the film below it, in the shape of a spherical cap, which is of the same order in size as the droplet itself. The deformations generated may be orders of magnitude larger than the bulk elastocapillary length, because stretching of the membrane is the relevant mode of elasticity [28-31]. The contact line profile is determined by a Neumann construction, which incorporates both mechanical and interfacial tensions. This profile is characterized by the angles subtended by the liquid $\left(\theta_{d}\right)$ and bulge $\left(\theta_{b}\right)$ to the surrounding film [Fig. 1(a)], which remains completely flat, i.e., the film's angle relative to the horizontal $\theta_{m}=0$. From the Neumann construction, these angles are set by two parameters: the Young's angle $\theta_{Y}$ of the same solid supported on a rigid substrate and the ratio $T_{\mathrm{in}} / \gamma$, where $T_{\text {in }}$ is the total mechanical and interfacial tension acting inside the contact region of the membrane or drop system [31]. In the limit of infinite tension, the bulge vanishes and Young's law is recovered.

In this study, we explore the partial wetting of a liquid droplet resting on an elastomeric membrane with an anisotropic tension. Surprisingly, the droplet assumes a shape which is elongated along the direction of high tension. We show from minimal theoretical considerations that the tensions in the film determine both the elongated shape of the wetting region and an observed out-of-plane deformation of the film surrounding the droplet. Therefore, liquid droplets serve as nondestructive probes for mapping out the stress field in a membrane.

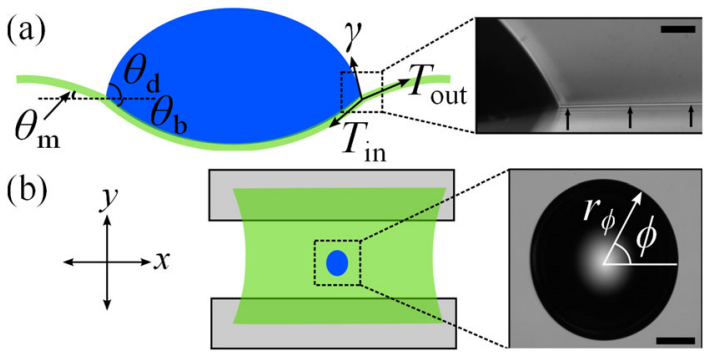

FIG. 1. (a) Schematic of the side view of the drop and membrane system. The contact angle profile is determined by the force balance shown. On the right, an optical image of the contact line region taken along the $x$ direction is shown. The dark part is the liquid, the lighter gray part below is simply a reflection of the droplet off the film, and the thin light curve on the right (indicated by arrows) is the film itself. (b) Schematic of a freestanding elastomeric film between two supports with a single droplet atop. An optical top view image of the droplet is shown on the right. Scale bars $=200 \mu \mathrm{m}$. 
Freestanding elastomeric films of thickness $h \sim 240 \mathrm{~nm}$ were prepared from Elastollan TPU 1185A (BASF) and suspended between two supports, as seen in Fig. 1(b) [32]. The sample is then stretched along the $y$ direction to produce a biaxial mechanical tension $T$, such that $T_{y}>T_{x}$. As can be seen in Fig. 1(b), since the edges in the $x$ direction of the film are free, the membrane assumes a bowed configuration. However, at the center of the film, far from the edges, there is a biaxial tension which is uniform. In an experiment, a droplet of glycerol is placed near the center of the film. In doing so, we find that the droplet assumes a noncircular footprint which is elongated along the high tension direction [Fig. 1(b)]. Initially, the contact line is seen to move as the droplet configuration equilibrates, indicating that there is no pinning in our system. Thus, we wait until the contact line reaches equilibrium ( $\sim 5 \mathrm{~min}$ ) before performing measurements. We advise that contact angle hysteresis should be minimized a priori so that equilibrium contact angles are indeed measured. Optical contact angle measurements are performed by viewing the sample from the side. These measurements are taken in two directions: viewing the sample from the $x$ direction, where the bulge and liquid-air interface can be simultaneously seen [as in Fig. 1(a)], and viewing the sample from the $y$ direction. When observing from the $y$ direction, the supports block the view of the lower side of the film; hence, two separate droplets are needed, one on the top side and one on the bottom side of the film, to get images of both the bulge and liquid-air interface. Images of the two droplets are also taken from above, from which we obtain precise measurements of the contact radii $r_{x}$ and $r_{y}$ and, hence, the aspect ratio $\epsilon \equiv r_{y} / r_{x}$. We use droplets with contact radii in the range of $300-450 \mu \mathrm{m}$, small enough that gravity does not play a role but large enough that evaporation does not influence our measurements over the experimental time scale. The droplets are much smaller than the overall lateral size of the film.

A sample optical image taken from the $x$ direction is shown in Fig. 2(a), where the droplet is sessile on top of the film, generating a bulge below it. The true 3D shape of the liquid-air interface is unknown. Nevertheless, its interface must have a constant mean curvature to ensure a constant

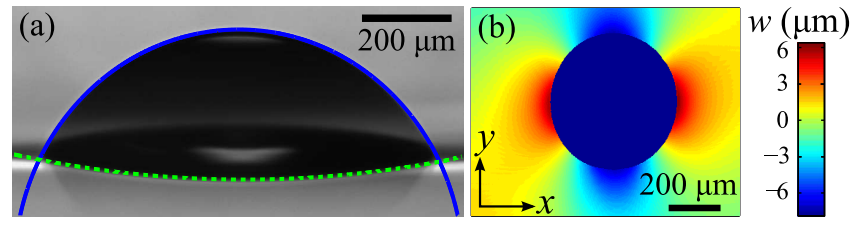

FIG. 2. (a) Optical side view taken from the $x$ direction. The solid curve is a circular fit to the liquid-air interface, and the dashed curve is a parabolic fit to the bulge. (b) Optical profilometry scan taken from above the droplet depicting the $z$ displacement $w$ of the film surrounding the wetting region (dark elliptical area). internal pressure. However, to simplify our analysis, we fit all liquid-air interface profiles to circular caps and extract the average radii of curvature $R_{d, x}$ and $R_{d, y}$. As can be seen by the solid curve in Fig. 2(a), these fits exhibit excellent agreement with our experimental images [32]. We note that $R_{d, y}>R_{d, x}$ in further support that the droplet's shape is not spherical. Knowing $r_{i}$ and $R_{i}$ in each direction $i=x, y$, the contact angles $\theta_{d, x}$ and $\theta_{d, y}$. [defined as the angles the liquid-air cap makes at the contact line relative to the $x-y$ plane as seen in Fig. 1(a)] can be determined. Furthermore, the bulge profiles are fit to parabolas, the justification for which will be provided later. As can be seen by the dashed curve in Fig. 2(a), we find these fits to capture the bulge profile well [32]. From these fits, we determine $\theta_{b, x}$ and $\theta_{b, y}$, defined as the angles the bulge makes at the contact line relative to the $x-y$ plane, as defined in Fig. 1(a).

We describe our films as membranes where bending can be ignored, and, hence, the membrane slope is thought of as being discontinuous at the contact line. In reality, there exists a narrow region near the contact line where bending dominates and the membrane curves to connect the bulge region to the outside region. However, since this bending region is too small to be measured in our experiments, the membrane description is appropriate. For droplets on membranes with isotropic tension, the film is flat outside the contact region, and the contact line shape is completely determined by $\theta_{d}$ and $\theta_{b}$ [28-31]. However, for films with anisotropic tension, the film experiences an out-of-plane deformation outside the wetting region. Therefore, a complete picture of the contact line profile must include the angle of the membrane relative to the $x-y$ plane at the contact line which we denote with $\theta_{m}$. As can be seen in Fig. 1(a), the membrane curves down towards the droplet in the $y$ direction, which we define to correspond to $\theta_{m}>0$. Conversely, the film curves up towards the droplet in the $x$ direction and $\theta_{m}<0$. Since $\left|\theta_{m}\right|$ is small $\left(<4^{\circ}\right)$ and the membrane is difficult to resolve optically, we employ optical profilometry (Veeco, Wyko NT1100) to probe the out-of-plane deformation of the membrane, $w$, where $w>0$ is defined to indicate the side from which the liquid droplet is placed. One such profilometry scan taken from the droplet side is shown in Fig. 2(b). The membrane is pulled towards positive $w$ on the low tension side while being displaced towards negative $w$ on the high tension side. From the profilometry data, it is straightforward to determine the values of $\theta_{m}$ in the two principal directions: $x$ and $y$.

Although the tension in the membrane is not known a priori, it may be determined using the contact line profile, as has been demonstrated in previous studies [28-31]. Through a Neumann construction, as depicted in Fig. 1(a), where $T_{\text {in }}$ and $T_{\text {out }}=T_{\text {in }}+\gamma \cos \theta_{Y}$ contain mechanical and interfacial tensions (see Ref. [31]), the contact line profile in a given direction is completely determined by the values of $T_{\text {in }} / \gamma$ and $\theta_{Y}$. The contact line profile is entirely 
TABLE I. Sample fit of contact angle data in the $x$ direction to a Neumann construction to extract $T_{\mathrm{in}, x} / \gamma=2.7 \pm 0.15$.

\begin{tabular}{lcc}
\hline \hline & Experiment & Best fit \\
\hline$\left(\theta_{d, x}+\theta_{m, x}\right)$ & $57.8 \pm 1.2^{\circ}$ & $59.5 \pm 0.6^{\circ}$ \\
$\left(\theta_{d, x}+\theta_{b, x}\right)$ & $76.6 \pm 1.1^{\circ}$ & $78.1 \pm 0.6^{\circ}$ \\
$\left(\theta_{b, x}-\theta_{m, x}\right)$ & $18.8 \pm 0.8^{\circ}$ & $18.7 \pm 1.1^{\circ}$ \\
\hline \hline
\end{tabular}

characterized by the three internal angles subtended at the contact line: $\pi-\theta_{d}-\theta_{m}, \theta_{d}+\theta_{b}$, and $\pi-\theta_{b}+\theta_{m}$. The Neumann construction is able to predict only these internal angles, not $\theta_{d}, \theta_{b}$, and $\theta_{m}$ individually. Therefore, using the value for $\theta_{Y}$ and the angles $\theta_{d}, \theta_{b}$, and $\theta_{m}$, we fit the internal angles to the Neumann construction prediction in $x$ and $y$ with $T_{\mathrm{in}, x} / \gamma$ and $T_{\mathrm{in}, y} / \gamma$ as the fitting parameters (see Ref. [32]). The best fit allows us to extract values of $T_{\mathrm{in}, x} / \gamma$ and $T_{\text {in, } y} / \gamma$. A sample fit is provided in Table 1 , where it is clear that a single value of $T_{\mathrm{in}, x} / \gamma$ captures the data well. The tensions measured in this way were found to be consistent with tensions computed from the strains in the film during stretching, attained using particle tracking in a separate experiment [32].

We now turn to theoretical considerations and begin with some simplifications to render the problem more tractable. First, we restrict our analysis to the case in which $\epsilon$ is close to unity, where the shape of the droplet may be treated as a perturbation to a spherical cap. As such, we make the assumption that the liquid-air interface profile as viewed along a general sight line oriented at $\phi$ [Fig. 1(b)] is simply a circular cap with its own radius of curvature. This assumption is validated by the fact that such profiles are well fitted to circular caps [32]. Similarly, as will be justified later, all profiles of the bulge are parabolic in a good approximation. We make the simplistic assumption that the deformations produced by the two droplets on the film are perturbative only to the pretension. This assumption was found to be valid in a previous study done with isotropic tension [31] but is further supported by the fact that the contact angles are unchanged as additional droplets are placed onto the film as well as by the tension measurement done using particle tracking [32]. Finally, we make small angle approximations when appropriate [37]. To predict the shape of the droplet's footprint, i.e., projection of the wetted region onto the $x-y$ plane, we make use of the fact that the total height from the bottom of the bulge to the top of the droplet must be equal in every profile. We find that the contact radius $r_{\phi}$ [defined in Fig. 1(b)] of the footprint is given by (see Ref. [32])

$$
r_{\phi}\left[\arccos \left(\cos \theta_{Y}-\frac{\gamma}{2 T_{\mathrm{in}, \phi}} \sin ^{2} \theta_{Y}\right)\right]=C,
$$

where $T_{\mathrm{in}, \phi}$ is the total (mechanical and interfacial) tension in the $\phi$ direction in the region under the droplet and $C$ is a
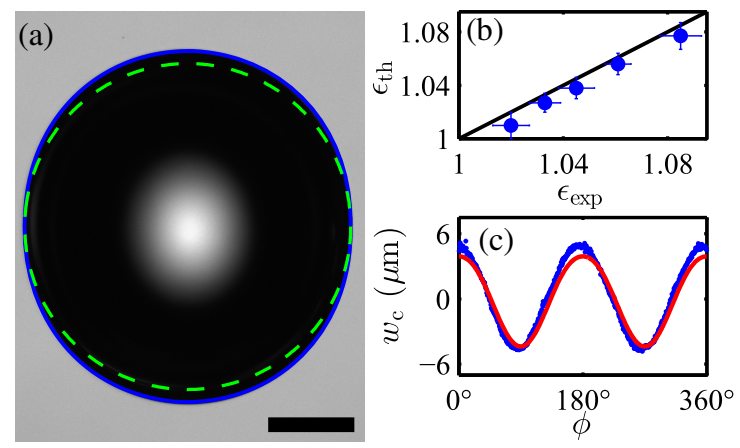

FIG. 3. (a) Top view image of an elongated droplet $(\epsilon \sim 1.09)$ where the solid curve represents the best fit of Eq. (1) and the dashed curve is a circle drawn for comparison. Scale bar $=200 \mu \mathrm{m}$. (b) A comparison between the experimental and theoretical values of $\epsilon$ for all samples. The line drawn represents $\epsilon_{\text {th }}=\epsilon_{\text {exp }}$. (c) Vertical position of the contact line around a sample droplet, where the points correspond to experimental data and the solid curve is calculated from Eqs. (1) and (3) with $A$ and $B$ obtained from bulge profile fits.

constant which simply sets the overall length scale. We see that $r_{\phi}$ increases with $T_{\mathrm{in}, \phi}$, consistent with the observation that droplets are elongated along the direction of highest tension. Since the membrane tensions are assumed to be unchanged by the addition of droplets, the tension remains purely biaxial with its principal axes aligned along $x$ and $y$, and $T_{\mathrm{in}, \phi}=T_{\mathrm{in}, x} \cos ^{2} \phi+T_{\mathrm{in}, y} \sin ^{2} \phi$ [38]. In Fig. 3(a), we show an optical top view of an elongated droplet on a film, where we also plot Eq. (1) as a solid curve with $C=514 \mu \mathrm{m}$ found by fitting. We see that Eq. (1) provides an excellent approximation of the elongated shape of the footprint. Furthermore, Eq. (1) can be used to determine $\epsilon$ without any free parameters. We see that the aspect ratio is high when $T_{\mathrm{in}, y}$ is large while retaining a small $T_{\mathrm{in}, x}$. For a quantitative comparison with our experimental observations, we refer to Fig. 3(b), where all measurements of the aspect ratio $\epsilon_{\exp }$ are plotted against their predicted values $\epsilon_{\text {th }}$, computed using Eq. (1) and the measured values of $T_{\mathrm{in}, x} / \gamma$ and $T_{\mathrm{in}, y} / \gamma$. We find a good agreement between the experimental and theoretical values of $\epsilon$. We note that $\epsilon_{\mathrm{th}}$ is systematically smaller than $\epsilon_{\text {exp }}$, which we attribute to the simplifications made in the theory.

To construct a full theoretical treatment of the membrane deformation, one may follow the approach laid out in articles by Davidovitch and co-workers, where the Föpplvon Kármán equations are solved in the limit of negligible bending contributions $[7,8,39]$. However, further simplifications can be made when the deformation of the membrane by the droplet does not notably modify the preexisting tension. Justified by our experimental observations, we have already made this assumption (in the claim that $T_{\mathrm{in}, \phi}$ remains biaxial) to simplify the description of our system. As such, the deflection of a membrane (w) carrying a uniform biaxial tension of $T_{\mathrm{in}, x}, T_{\mathrm{in}, y}$ is given by $[32,40]$ 

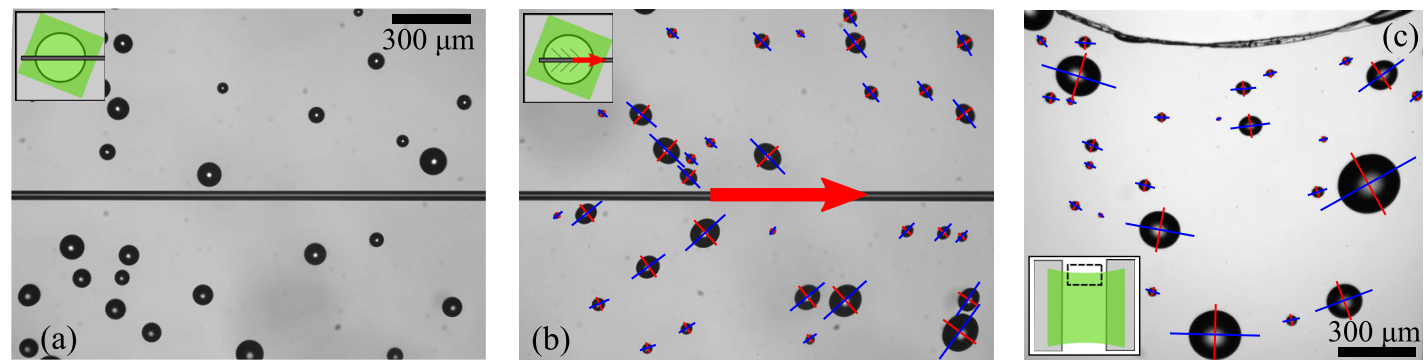

FIG. 4. (a) Droplets on a film with isotropic tension with a pipette laid across the film (schematized in the inset). (b) The pipette is moved in the direction indicated by the arrow to shear the film (schematized in the inset, where the thin diagonal lines indicate the principal direction of high tension). (c) Droplets deposited near the boundary (see the inset) of a film. The major (minor) axis of the droplets are indicated by a long (short) line in (b) and (c).

$$
T_{\mathrm{in}, x} \frac{\partial^{2} w}{\partial x^{2}}+T_{\mathrm{in}, y} \frac{\partial^{2} w}{\partial y^{2}}=-p(x, y)
$$

where $p$ is the pressure distribution acting on the film. This equation is essentially Laplace's law but with anisotropic tension and in the limit of small membrane slopes. The small slope approximation is appropriate in this case, since the bulge contact angles are always below $25^{\circ}$. From this equation, it is straightforward to solve for the shape of the bulge, which forms in response to the uniform Laplace pressure over the wetted region. We propose a solution of the form

$$
w=A x^{2}+B y^{2}+w_{0},
$$

where $w_{0}$ is an arbitrary vertical shift and $A$ and $B$ are found experimentally [32]. Thus, any vertical cross section of the bulge passing through its apex is simply a parabola, which is the reason for fitting the bulge profiles with parabolas to extract $\theta_{b, x}$ and $\theta_{b, y}$. From these parabolic fits, the values of $A$ and $B$ are determined. Of course, we must still ensure Eq. (2) is satisfied. Substituting $w$ back into Eq. (2) generates a criterion which can be written as [32]

$$
\frac{T_{\mathrm{in}, x}}{\gamma} \theta_{b, x}+\frac{T_{\mathrm{in}, y}}{\gamma \epsilon} \theta_{b, y}=\sin \theta_{d, x}+\frac{1}{\epsilon} \sin \theta_{d, y} .
$$

Evaluating both sides of Eq. (4) using all our data yields a constant value of $1.75 \pm 0.03$ for the left side and $1.70 \pm$ 0.06 for the right side, indicating that the data and theory are consistent.

A striking aspect of the system is the out-of-plane deformation of the membrane surrounding the wetting region, as evidenced in Fig. 2(b). As shown for a sample droplet in Fig. 3(c), the vertical position $w_{c}$ of the contact line relative to its average value oscillates with $\phi$. Knowing all parameters in Eqs. (1) and (3), we may attain a prediction for $w_{c}$ by evaluating Eq. (3) at the position of the contact line given by Eq. (1). Therefore, we plot the prediction of the data in Fig. 3(c) with a solid curve and find that it exhibits excellent agreement with the data.
As we have seen, droplets are elongated along the high tension direction; i.e., the major and minor axes of the droplets' footprints align with the principal tension directions in the membrane [38]. We can further test this property by a separate experiment where samples are made with different stress fields in which the principal directions are known. As shown in Fig. 4(a), water droplets have been sprayed onto a freestanding elastomeric film on a circular washer, where the tension is completely isotropic and uniform. A thin glass pipette has then been placed into contact across the film but does not significantly modify the film's stresses. As such, the droplets are completely round, in agreement with previous work done with isotropic tension [31]. After these droplets have evaporated, we displace the pipette slightly towards the right, as seen in Fig. 4(b), which generates a shear stress in the membrane. If a membrane with isotropic stress is subjected to a shear stress $\tau_{x y}$, the principal directions of the stress are aligned at $45^{\circ}$ to the $x$ and $y$ axes regardless of the shear's magnitude [38]. To employ this known stress field as a test, we spray water droplets onto the film and fit the footprints of these with ellipses to extract the major (minor) axis, which is displayed in Figs. 4(b), 4(c) as a long (short) line. Computing the average angle that the major axes subtend to the pipette, we find $44 \pm 8^{\circ}$, as we would expect. Finally, at a free (i.e., stress-free) boundary, the principal directions are tangent (high tension) and normal (low tension) to the boundary [38]. We thus purposely deposit liquid droplets near the bowed edges [see Fig. 1(b)] of our stretched sample, as seen in Fig. 4(c). Indeed, the major and minor axes plotted atop the droplets align with the principal directions. Note that, although we do not observe any mutual influence between droplets, we expect such an effect to become important at sufficiently small separations.

In this work, the droplets' elongated shape stems from the anisotropy in the compliance of the freestanding film in the two orthogonal directions. On the other hand, it is known that droplets atop stretched substrates which are noncompliant in the out-of-plane direction may also experience anisotropic wetting conditions and become 
elongated [41-43]. This is a result of two effects: anisotropic molecular forces or compositions caused by stretching the molecules at the surface [41,42] or an induced anisotropy in the topographical roughness $[43,44]$. These effects do not contribute to the results within our study, since droplets are round when placed atop stretched films which have been transferred onto a rigid substrate.

We have performed experiments studying liquid droplets atop deformable membranes which carry an anisotropic tension. Droplets assume shapes which deviate from spherical caps and become elongated along the direction of highest tension. By measuring the contact line profile, we completely determine the tensions in the membrane. Using these tensions, along with a minimal theoretical model, we are able to form accurate predictions for the elongated shape of the droplet's footprint and the out-ofplane deformation of the membrane surrounding this region. Thus, liquid droplets may be used as a tool to map out the magnitudes and directions of the stresses in a membrane-analogous to iron filings in magnetic fields.

The financial support by Natural Science and Engineering Research Council of Canada and the Global Station for Soft Matter, a project of Global Institution for Collaborative Research and Education at Hokkaido University, is gratefully acknowledged.

*dalnoki@mcmaster.ca

[1] C. Py, P. Reverdy, L. Doppler, J. Bico, B. Roman, and C. N. Baroud, Phys. Rev. Lett. 98, 156103 (2007).

[2] N. Patra, B. Wang, and P. Král, Nano Lett. 9, 3766 (2009).

[3] J. van Honschoten, J. Berenschot, T. Ondarcuhu, R. Sanders, J. Sundaram, M. Elwenspoek, and N. Tas, Appl. Phys. Lett. 97, 014103 (2010).

[4] J. Bae, T. Ouchi, and R. C. Hayward, ACS Appl. Mater. Interfaces 7, 14734 (2015).

[5] J. Huang, M. Juszkiewicz, W. H. de Jeu, E. Cerda, T. Emrick, N. Menon, and T. P. Russell, Science 317, 650 (2007).

[6] D. Vella, M. Adda-Bedia, and E. Cerda, Soft Matter 6, 5778 (2010).

[7] H. King, R. D. Schroll, B. Davidovitch, and N. Menon, Proc. Natl. Acad. Sci. U.S.A. 109, 9716 (2012).

[8] R. D. Schroll, M. Adda-Bedia, E. Cerda, J. Huang, N. Menon, T.P. Russell, K. B. Toga, D. Vella, and B. Davidovitch, Phys. Rev. Lett. 111, 014301 (2013).

[9] B. Andreotti, O. Baumchen, F. Boulogne, K. E. Daniels, E. R. Dufresne, H. Perrin, T. Salez, J. H. Snoeijer, and R. W. Style, Soft Matter 12, 2993 (2016).

[10] B. Roman and J. Bico, J. Phys. Condens. Matter 22, 493101 (2010).

[11] M. Rivetti and S. Neukirch, Proc. R. Soc. A 468, 1304 (2012).

[12] A. Fargette, S. Neukirch, and A. Antkowiak, Phys. Rev. Lett. 112, 137802 (2014).
[13] H. Elettro, F. Vollrath, A. Antkowiak, and S. Neukirch, Int. J. Nonlinear Mech. 75, 59 (2015).

[14] A. Sauret, F. Boulogne, K. Somszor, E. Dressaire, and H. A. Stone, Soft Matter 13, 134 (2017).

[15] H. Elettro, S. Neukirch, F. Vollrath, and A. Antkowiak, Proc. Natl. Acad. Sci. U.S.A. 113, 6143 (2016).

[16] R. D. Schulman, A. Porat, K. Charlesworth, A. Fortais, T. Salez, E. Raphaël, and K. Dalnoki-Veress, Soft Matter 13, 720 (2017).

[17] R. W. Style et al., Proc. Natl. Acad. Sci. U.S.A. 110, 12541 (2013).

[18] S. Karpitschka, A. Pandey, L. A. Lubbers, J. H. Weijs, L. Botto, S. Das, B. Andreotti, and J. H. Snoeijer, Proc. Natl. Acad. Sci. U.S.A. 113, 7403 (2016).

[19] T. Liu, X. Xu, N. K. Nadermann, Z. He, A. Jagota, and C.-Y. Hui, Langmuir 33, 75 (2017).

[20] R. Pericet-Cámara, A. Best, H. J. Butt, and E. Bonaccurso, Langmuir 24, 10565 (2008).

[21] E. R. Jerison, Y. Xu, L. A. Wilen, and E. R. Dufresne, Phys. Rev. Lett. 106, 186103 (2011).

[22] R. W. Style and E. R. Dufresne, Soft Matter 8, 7177 (2012).

[23] R. W. Style, R. Boltyanskiy, Y. Che, J. S. Wettlaufer, L. A. Wilen, and E. R. Dufresne, Phys. Rev. Lett. 110, 066103 (2013)

[24] A. Marchand, S. Das, J. H. Snoeijer, and B. Andreotti, Phys. Rev. Lett. 109, 236101 (2012).

[25] S. J. Park, B. M. Weon, J. S. Lee, J. Lee, J. Kim, and J. H. Je, Nat. Commun. 5, 4369 (2014).

[26] C.-Y. Hui and A. Jagota, Proc. R. Soc. A 470 (2014).

[27] M. E. Shanahan, J. Adhes. 18, 247 (1985).

[28] N. Nadermann, C.-Y. Hui, and A. Jagota, Proc. Natl. Acad. Sci. U.S.A. 110, 10541 (2013).

[29] C.-Y. Hui, A. Jagota, N. Nadermann, and X. Xu, Procedia IUTAM 12, 116 (2015).

[30] C.-Y. Hui and A. Jagota, Soft Matter 11, 8960 (2015).

[31] R. D. Schulman and K. Dalnoki-Veress, Phys. Rev. Lett. 115, 206101 (2015).

[32] See Supplemental Material at http://link.aps.org/ supplemental/10.1103/PhysRevLett.118.198002 for a more detailed overview of the sample preparation, optical profiles of a droplet and bulge viewed along the y direction, an explanation of the particle tracking experiment to verify measured tensions, the theoretical arguments leading to Eqs. (1), (3), and (4), the Neumann construction used in the fits, and Refs. [33-36].

[33] R. Pan and D. Wati, Polymer Composites 17, 486 (1996).

[34] P. Russo, M. Lavorgna, F. Piscitelli, D. Acierno, and L. Di Maio, Eur. Polym. J. 49, 379 (2013).

[35] H.-Y. Mi, X. Jing, M. R. Salick, W. C. Crone, X.-F. Peng, and L.-S. Turng, Adv. Polym. Tech. 33, 21380 (2014).

[36] D. R. Lide, CRC Handbook of Chemistry and Physics (CRC Press, Boca Raton, FL, 2004), pp. 6-154.

[37] In the theoretical model and we make the approximation that $\tan \theta_{b} \approx \theta_{b}$ and $\tan \theta_{d} / 2 \approx \theta_{d} / 2$. In our experiments, $\theta_{b}<25^{\circ}$ and $\theta_{d} / 2<33^{\circ}$, so there is less than $\sim 10 \%$ error in making these approximations.

[38] S. Timoshenko and J. Goodier, Theory of Elasticity, 2nd ed. (McGraw-Hill, New York, 1951). 
[39] B. Davidovitch, R. D. Schroll, D. Vella, M. Adda-Bedia, and E. A. Cerda, Proc. Natl. Acad. Sci. U.S.A. 108, 18227 (2011).

[40] L. Landau and E. Lifshitz, Theory of Elasticity, 3rd ed. (Butterworth-Heinemann, New York, 1986).

[41] R. J. Good, J. A. Kvikstad, and W. O. Bailey, J. Colloid Interface Sci. 35, 314 (1971).
[42] E. H. Cirlin and D. H. Kaelble, J. Polym. Sci., Polym. Phys. Ed. 11, 785 (1973).

[43] N. Sung, H. Lee, P. Yuan, and C. Sung, Polym. Eng. Sci. 29, 791 (1989).

[44] H. Schonhorn, J. Adhes. 23, 147 (1987). 